\title{
A lower bound on the average degree forcing a minor
}

\author{
Sergey Norin* \\ Department of Mathematics and Statistics \\ McGill University \\ Montréal, Canada \\ snorin@math.mcgill.ca
}

\author{
Bruce Reed \\ School of Computer Science \\ McGill University \\ Montréal, Canada \\ breed@cs.mcgill.ca
}

\author{
Andrew Thomason \\ Department of Pure Mathematics and Mathematical Statistics \\ University of Cambridge \\ United Kingdom \\ A.G.Thomason@dpmms.cam.ac.uk \\ David R. Wood ${ }^{\dagger}$ \\ School of Mathematics \\ Monash University \\ Melbourne, Australia \\ david.wood@monash.edu
}

Submitted: Jul 7, 2019; Accepted: Mar 9, 2020; Published: Apr 3, 2020

(C) The authors. Released under the CC BY-ND license (International 4.0).

\begin{abstract}
We show that for sufficiently large $d$ and for $t \geqslant d+1$, there is a graph $G$ with average degree $(1-\varepsilon) \lambda t \sqrt{\ln d}$ such that almost every graph $H$ with $t$ vertices and average degree $d$ is not a minor of $G$, where $\lambda=0.63817 \ldots$ is an explicitly defined constant. This generalises analogous results for complete graphs by Thomason (2001) and for general dense graphs by Myers and Thomason (2005). It also shows that an upper bound for sparse graphs by Reed and Wood (2016) is best possible up to a constant factor.
\end{abstract}

Mathematics Subject Classifications: 05C83; 05C35

*Norin is supported by NSERC grant 418520 .

${ }^{\dagger}$ Wood is supported by the Australian Research Council. 


\section{Introduction}

Mader [20] first proved that for every graph $H$, every graph with sufficiently large average degree contains $H$ as a minor ${ }^{1}$. The natural extremal question arises: what is the least average degree that forces $H$ as a minor? To formalise this question, let $f(H)$ be the infimum of all real numbers $d$ such that every graph with average degree at least $d$ contains $H$ as a minor. This value has been extensively studied for numerous graphs $H$, including small complete graphs [6, 12, 21, 28, 29], the Petersen graph [11], general complete graphs $[2,5,14,15,21,23,30,31]$, complete bipartite graphs [3, 16-19, 24], general dense graphs [25], general sparse graphs [9, 26, 27], disjoint unions of graphs [4, 13, 33], and disjoint unions of cycles [8]; see [32] for a survey.

For complete graphs $K_{t}$, the above question was asymptotically answered in the following theorem of Thomason [31], where

$$
\lambda:=\max _{x>0} \frac{1-e^{-x}}{\sqrt{x}}=0.63817 \ldots
$$

Theorem 1 ([31]). Every graph with average degree at least $(\lambda+o(1)) t \sqrt{\ln t}$ contains $K_{t}$ as a minor. Conversely, there is a graph with average degree at least $(\lambda+o(1)) t \sqrt{\ln t}$ that contains no $K_{t}$ minor. That is,

$$
f\left(K_{t}\right)=(\lambda+o(1)) t \sqrt{\ln t} .
$$

Myers and Thomason [25] generalised this result for all families of dense graphs as follows ${ }^{2}$.

Theorem 2 ([25]). For every $\tau \in(0,1)$, for all $t$ and $d \geqslant t^{\tau}$, for almost every graph $H$ with $t$ vertices and average degree $d$ (and for every d-regular graph with $t$ vertices),

$$
f(H)=(\lambda+o(1)) t \sqrt{\ln d}
$$

Theorem 2 determines $f(H)$ for most dense graphs $H$ with $d \geqslant t^{\tau}$, but says nothing for sparse graphs $H$, where $d$ can be much smaller than $t$. In this regime, Reed and Wood $[26,27]$ established the following upper bound on $f(H)$.

Theorem 3 ([26, 27]). For sufficiently large $d$, and for every graph $H$ with $t$ vertices and average degree $d$, every graph with average degree at least $3.895 t \sqrt{\ln d}$ contains $H$ as a minor. That is,

$$
f(H) \leqslant 3.895 t \sqrt{\ln d}
$$

\footnotetext{
${ }^{1} \mathrm{~A}$ graph $H$ is a minor of a graph $G$ if a graph isomorphic to $H$ can be obtained from a subgraph of $G$ by contracting edges.

${ }^{2}$ As is standard, we write that almost every graph with $t$ vertices and average degree $d$ satisfies property $P$ if the probability that a random graph with $t$ vertices and average degree $d$ satisfies property $P$ tends to 1 as $t \rightarrow \infty$.
} 
The purpose of this paper is to show that this result is best possible up to a constant factor. Indeed, we precisely match the lower bounds in the work of Thomason [31] and Myers and Thomason [25], strengthening the lower bound in Theorem 2 by eliminating the assumption that $d \geqslant t^{\tau}$. Informally, we prove that if $d$ is large, then almost every $H$ with $t \geqslant d+1$ vertices and average $d$ satisfies $f(H) \geqslant(1-\varepsilon) \lambda t \sqrt{\ln d}$. To state the result precisely, let $\mathcal{G}(t, m)$ be the space of random graphs with vertex-set $\{1,2, \ldots, t\}$ and $m$ edges. Thus $\mathcal{G}(t, t d / 2)$ is the space of random graphs with $t$ vertices and average degree $d$.

Theorem 4. For every $\varepsilon, c \in(0,1)$ there exists $d_{0}$ such that for every integer $d \geqslant d_{0}$ and for every integer $t \geqslant d+1$, there is a graph $G$ with average degree at least $(1-\varepsilon) \lambda t \sqrt{\ln d}$ such that if $H \in \mathcal{G}(t, t d / 2)$ then $\mathbb{P}(H$ is a minor of $G)<c^{t}$, and in particular, $\mathbb{P}(f(H) \geqslant$ $(1-\varepsilon) \lambda t \sqrt{\ln d})>1-c^{t}$.

Note that in the proofs of Theorems 1 and 2 the host graph $G$ is a random graph of appropriately chosen constant density. Indeed, every such extremal graph is essentially a disjoint union of pseudo-random graphs $[23,25]$. However, random graphs themselves are not extremal when $d$ is small compared to $t$. Indeed, Alon and Füredi [1] showed that if $d \leqslant \log _{2} t$ then, for every graph $H$ with $t$ vertices and maximum degree $d$, a random graph on $t$ vertices (with edge probability $\frac{1}{2}$ ) will almost certainly contain a spanning copy of $H$. To prove Theorem 4, we take $G$ to be a blowup of a suitably chosen small random graph. Note that Fox [7] also considers minors of blowups of random graphs. On the face of it, such blowups might not to be pseudo-random, thus contradicting the fact that in many cases the extremal graphs are known to be pseudo-random. But the notion of pseudo-randomness involved is weak, asserting only that induced subgraphs of constant proportion have roughly the same density, and the blowups used here have this property.

Note that Reed and Wood [26] claimed that a lower bound analogous to Theorem 4 followed from the work of Myers and Thomason [25]. However, this claim is invalid. The error occurs in the footnote on page 302 of [26], where Theorem 4.8 and Corollary 4.9 of Myers and Thomason [25] are applied. The assumptions in these results mean that they are only applicable if the average degree of $H$ is at least $|V(H)|^{\varepsilon}$ for some fixed $\varepsilon>0$, which is not the case here. Also note that Reed and Wood [26] claimed that a $c t \sqrt{\log d}$ lower bound holds for every $d$-regular graph (also as a corollary of the work of Myers and Thomason [25]). This is false, for example, when $H$ is the $d$-dimensional hypercube [10].

\section{The Proof}

We will need the following Chernoff Bound.

Lemma $5([22])$. Let $X_{1}, X_{2}, \ldots, X_{n}$ be independent random variables, where each $X_{i}=1$ with probability $p$ and $X_{i}=0$ with probability $1-p$. Let $X:=\sum_{i=1}^{n} X_{i}$. Then for $\delta \in(0,1)$,

$$
\mathbb{P}(X \leqslant(1-\delta) p n) \leqslant \exp \left(-\frac{\delta^{2}}{2} p n\right) .
$$

Let $G$ be a graph. For $\ell \in \mathbb{R}^{+}$, a non-empty set of at most $\ell$ vertices in $G$ is called an $\ell$-set. Two sets $A$ and $B$ of vertices in $G$ are non-adjacent if there is no edge in $G$ between $A$ and $B$. 
Our first lemma gives properties about a random graph.

Lemma 6. Fix $p, \varepsilon, \alpha \in(0,1)$ and $\beta \in(\alpha, 1)$, and let $b:=(1-p)^{-1}$. Then there exists $d_{0}$ such that for every integer $d \geqslant d_{0}$, if $s:=\left\lceil d^{\beta}\right\rceil$ and $\ell:=\sqrt{\alpha \log _{b} d}$, then there exists a graph $G$ with exactly $d$ vertices and more than $\left(\frac{1}{2}-\varepsilon\right) p d^{2}$ edges, such that for every set $S$ of s pairwise disjoint $\ell$-sets in $G$, more than $\frac{1}{2} d^{-\alpha}\left(\begin{array}{l}s \\ 2\end{array}\right)$ pairs of $\ell$-sets in $S$ are non-adjacent.

Proof. Let $G$ be a graph on $d$ vertices, where each edge is chosen independently at random with probability $p$. By Lemma 5 , the probability that $|E(G)| \leqslant\left(\frac{1}{2}-\varepsilon\right) p d^{2}$ is less than $\frac{1}{2}$.

If $A$ and $B$ are disjoint $\ell$-sets, then the probability that $A$ and $B$ are non-adjacent equals $(1-p)^{|A||B|} \geqslant(1-p)^{\ell^{2}}=d^{-\alpha}$. Consider a set $S$ of $s$ pairwise disjoint $\ell$-sets in $G$. Let $X_{S}$ be the number of pairs of elements of $S$ that are non-adjacent. Since the elements of $X_{S}$ are pairwise disjoint, Lemma 5 is applicable and implies that the probability that $X_{S} \leqslant \frac{1}{2} d^{-\alpha}\left(\begin{array}{l}s \\ 2\end{array}\right)$ is at most $\exp \left(-\frac{1}{8} d^{-\alpha}\left(\begin{array}{l}s \\ 2\end{array}\right)\right) \leqslant \exp \left(-\frac{1}{16} d^{\beta-\alpha}(s-1)\right)$, which is at most $\frac{1}{2}\left(2 d^{\ell}\right)^{-s}$ since $d$ is sufficiently large.

The number of $\ell$-sets is $\sum_{i=1}^{\ell}\left(\begin{array}{l}d \\ i\end{array}\right) \leqslant 2 d^{\ell}$. Thus the number of sets of $s$ pairwise disjoint $\ell$-sets is at most $\left(\begin{array}{c}2 d^{\ell} \\ s\end{array}\right) \leqslant\left(2 d^{\ell}\right)^{s}$. By the union bound, the probability that $X_{S} \leqslant \frac{1}{2} d^{-\alpha}\left(\begin{array}{l}s \\ 2\end{array}\right)$, for some set $S$ of $s$ pairwise disjoint $\ell$-sets, is less than $\frac{1}{2}$.

Hence with positive probability, $|E(G)|>\left(\frac{1}{2}-\varepsilon\right) p d^{2}$ edges, and $X_{S}>\frac{1}{2} d^{-\alpha}\left(\begin{array}{l}s \\ 2\end{array}\right)$ for every set $S$ of $s$ pairwise disjoint $\ell$-sets. The result follows.

The next lemma is the heart of our proof.

Lemma 7. Fix $p, \varepsilon, \alpha, c \in(0,1)$ and let $b:=(1-p)^{-1}$. Then there exists $d_{0}$ such that for every integer $d \geqslant d_{0}$ and for every integer $t \geqslant d+1$, there is a graph $G$ with average degree at least $(1-\varepsilon) p t \sqrt{\alpha \log _{b} d}$ such that such that if $H \in \mathcal{G}(t, t d / 2)$ then $\mathbb{P}(H$ is a minor of $G)<c^{t}$.

Proof. Let $\ell:=\sqrt{\alpha \log _{b} d}$. Choose $\beta \in(\alpha, 1)$ and let $s:=\left\lceil d^{\beta}\right\rceil$. We assume that $d$ is sufficiently large as a function of $\alpha, \beta$ and $\varepsilon$ to satisfy the inequalities occurring throughout the proof.

Let $G_{0}$ be the graph from Lemma 6 applied with $\frac{\varepsilon}{4}$ in place of $\varepsilon$. Thus $\left|V\left(G_{0}\right)\right|=d$ and $\left|E\left(G_{0}\right)\right|>\left(\frac{1}{2} \frac{\varepsilon}{4}\right) p d^{2}=\frac{1}{2}\left(1-\frac{\varepsilon}{2}\right) p d^{2}$, and for every set $S$ of $s$ pairwise disjoint $\ell$-sets in $G_{0}$, more than $\frac{1}{2} d^{-\alpha}\left(\begin{array}{l}s \\ 2\end{array}\right)$ pairs of $\ell$-sets in $S$ are non-adjacent. Call this property $(\star)$.

Let $G$ be obtained from $G_{0}$ by replacing each vertex $x$ by an independent set $I_{x}$ of size

$$
r:=\left\lceil\left(1-\frac{\varepsilon}{2}\right) \frac{t \ell}{d}\right\rceil,
$$

and replacing each edge $x y$ of $G_{0}$ by a complete bipartite graph between $I_{x}$ and $I_{y}$. Note that

$$
|V(G)|=d r=d\left\lceil\left(1-\frac{\varepsilon}{2}\right) \frac{t \ell}{d}\right\rceil<\left(1-\frac{\varepsilon}{4}\right) \ell t,
$$

and

$$
|E(G)|=\left|E\left(G_{0}\right)\right| r^{2}>\left(1-\frac{\varepsilon}{2}\right) \frac{p r^{2} d^{2}}{2}=\left(1-\frac{\varepsilon}{2}\right) \frac{p r d}{2}|V(G)| \geqslant\left(1-\frac{\varepsilon}{2}\right)^{2} \frac{p t \ell}{2}|V(G)| .
$$


Hence $G$ has average degree $2 \frac{|E(G)|}{|V(G)|} \geqslant\left(1-\frac{\varepsilon}{2}\right)^{2} p t \ell \geqslant(1-\varepsilon) p t \sqrt{\alpha \log _{b} d}$, as claimed. It remains to show that almost every graph $H$ with $t$ vertices and average degree $d$ is not a minor of $G$.

A blob is a non-empty subset of $V\left(G_{0}\right)$. A blobbing $\left(B_{1}, B_{2}, \ldots, B_{t}\right)$ is an ordered sequence of $t$ blobs with total size at most $|V(G)|$, such that each vertex of $G_{0}$ is in at most $r$ blobs.

The motivation for these definitions is as follows: Suppose that a graph $H$ is a minor of $G$ and $V(H)=\{1,2, \ldots, t\}$. Then for each vertex $v$ of $H$ there is a set $X_{v} \subseteq V(G)$, such that $X_{v} \cap X_{w}=\emptyset$ for distinct $v, w \in V(H)$, and for every edge $v w$ of $H$, there is an edge in $G$ between $X_{v}$ and $X_{w}$. For each vertex $v$ of $H$, let $B_{v}:=\left\{x \in V\left(G_{0}\right): X_{v} \cap I_{x} \neq \emptyset\right\}$, called the projection of $X_{v}$ to $G_{0}$. Note that $\sum_{v}\left|B_{v}\right| \leqslant \sum_{v}\left|X_{v}\right| \leqslant|V(G)|$, and each vertex of $G_{0}$ is in at most $r$ of $B_{1}, B_{2}, \ldots, B_{t}$. Thus $\left(B_{1}, B_{2}, \ldots, B_{t}\right)$ is a blobbing. Also note that by the construction of $G$, if $B_{v} \cap B_{w}=\emptyset$, then there is an edge of $G$ between $X_{v}$ and $X_{w}$ if and only if there is an edge of $G_{0}$ between $B_{v}$ and $B_{w}$.

Claim 1. The number of blobbings is at most $(4 d)^{t \ell}$.

Proof. For positive integers $d, t$ and for each positive integer $n \geqslant d$, let $g(d, t, n)$ be the number of $t$-tuples $\left(X_{1}, X_{2}, \ldots, X_{t}\right)$ such that $X_{i}$ is a non-empty subset of $\{1,2, \ldots, d\}$ for all $i \in\{1,2, \ldots, t\}$, and $\sum_{i=1}^{t}\left|X_{i}\right| \leqslant n$. Below we prove that $g(d, t, n) \leqslant(4 d)^{n}$ by induction on $t$. The result follows, since the number of blobbings is at most $g(d, t,|V(G)|) \leqslant g(d, t,\lfloor t \ell\rfloor)$.

In the base case, $g(d, 1, n) \leqslant 2^{d} \leqslant(4 d)^{n}$, as desired. Now assume the claim for $t-1$. Observe that

$$
g(d, t, n)=\sum_{i=1}^{d}\left(\begin{array}{l}
d \\
i
\end{array}\right) g(d, t-1, n-i) .
$$

By induction,

$$
g(d, t, n) \leqslant \sum_{i=1}^{d}\left(\begin{array}{l}
d \\
i
\end{array}\right)(4 d)^{n-i} \leqslant \sum_{i=1}^{d}\left(\frac{e d}{i}\right)^{i}(4 d)^{n-i}=(4 d)^{n} \sum_{i=1}^{d}\left(\frac{e}{4 i}\right)^{i}<(4 d)^{n} .
$$

This completes the proof.

Two blobs are a good pair if they are disjoint and non-adjacent $\ell$-sets in $G_{0}$.

Claim 2. Every blobbing has at least $\frac{\varepsilon^{2}}{400} d^{-\alpha} t^{2}$ good pairs.

Proof. Suppose for a contradiction that some blobbing $\left(B_{1}, B_{2}, \ldots, B_{t}\right)$ has less than $\frac{\varepsilon^{2}}{400} d^{-\alpha} t^{2}$ good pairs. Let $X$ be the set of blobs $B_{i}$ such that $\left|B_{i}\right| \leqslant \ell$. Then $\ell(t-$ $|X|)<|V(G)|<\left(1-\frac{\varepsilon}{4}\right) \ell t$, implying $|X|>\frac{\varepsilon}{4} t$. Let $Y$ be the set of blobs in $X$ that belong to at most $\frac{\varepsilon}{20} d^{-\alpha} t$ good pairs. Thus the total number of good pairs is at least $\frac{\varepsilon}{40} d^{-\alpha} t|X \backslash Y|$, implying that $|X \backslash Y|<\frac{\varepsilon}{10} t$ and $|Y|>\frac{3 \varepsilon}{20} t$. Let $Z$ be a maximal subset of $Y$ such that the blobs in $Z$ are pairwise disjoint and contain at most $\frac{1}{2} d^{-\alpha}\left(\begin{array}{c}|Z| \\ 2\end{array}\right)$ good pairs. Then $1 \leqslant|Z|<s$ by property $(\star)$ of $G_{0}$. Let $Z^{\prime}$ be the set of blobs in $Y$ that are disjoint from every blob in $Z$. Since each blob in $Z$ intersects at most $\ell r$ other blobs, 
$|Y| \leqslant\left|Z^{\prime}\right|+\ell r|Z|<\left|Z^{\prime}\right|+\ell r s$, and $\left|Z^{\prime}\right|>\frac{3 \varepsilon}{20} t-\ell r s \geqslant \frac{\varepsilon}{10} t$ for sufficiently large $d$. By the maximality of $Z$, every blob in $Z^{\prime}$ is in a good pair with at least $\frac{1}{2} d^{-\alpha}|Z|$ blobs in $Z$. So in total there are at least $\frac{1}{2} d^{-\alpha}|Z|\left|Z^{\prime}\right|$ good pairs $\left\{B_{i}, B_{j}\right\}$ with $B_{i} \in Z$ and $B_{j} \in Z^{\prime}$. So some $B_{i} \in Z$ is in more than $\frac{1}{2} d^{-\alpha}\left|Z^{\prime}\right|>d^{-\alpha} \frac{\varepsilon}{20} t \operatorname{good}$ pairs, contradicting the definition of $Y$.

Let $H$ be a graph with $V(H)=\{1, \ldots, t\}$. We say that a blobbing $\left(B_{1}, B_{2}, \ldots, B_{t}\right)$ is $H$-compatible if for every $i j \in E(H)$ the blobs $B_{i}$ and $B_{j}$ intersect or are adjacent, implying that $\left\{B_{i}, B_{j}\right\}$ is not good. As explained above, if $H$ is a minor of $G$, then there exists an $H$-compatible blobbing. By Theorem 2, if $H \in \mathcal{G}(t, m)$ then the probability that a given blobbing is $H$-compatible is at most

$$
\left(\begin{array}{c}
\left(\begin{array}{c}
t \\
2
\end{array}\right)-\frac{\varepsilon^{2}}{400} d^{-\alpha} t^{2} \\
m
\end{array}\right) /\left(\begin{array}{c}
t \\
2 \\
m
\end{array}\right) \leqslant\left(1-\frac{\varepsilon^{2} d^{-\alpha}}{200}\right)^{m} \leqslant \exp \left(-\frac{\varepsilon^{2} m d^{-\alpha}}{200}\right) .
$$

Combining this inequality, Theorem 1 and the union bound, if $H \in \mathcal{G}(t, t d / 2)$ then

$$
\mathbb{P}(H \text { is a minor of } G) \leqslant(4 d)^{t \ell} \exp \left(-\frac{\varepsilon^{2} t d^{1-\alpha}}{400}\right)
$$

which is less than $c^{t}$ for sufficiently large $d$.

Proof of Theorem 4. Choose $x>0$ so that $\lambda=\frac{1-e^{-x}}{\sqrt{x}}$, let $b:=e^{x}, p:=1-e^{-x}$. Let $\alpha:=\left(\frac{1-\varepsilon}{1-\varepsilon / 2}\right)^{2}$, implying $\left(1-\frac{\varepsilon}{2}\right) \sqrt{\alpha}=1-\varepsilon$. By Lemma 7 , there exists $d_{0}$ such that for every integer $d \geqslant d_{0}$ and for every integer $t \geqslant d+1$, there is a graph $G$ with average degree at least $\left(1-\frac{\varepsilon}{2}\right) p t \sqrt{\alpha \log _{b} d}$ such that if $H \in \mathcal{G}(t, t d / 2)$ then $\mathbb{P}(H$ is a minor of $G)<c^{t}$. Since

$$
\left(1-\frac{\varepsilon}{2}\right) p t \sqrt{\alpha \log _{b} d}=\left(1-\frac{\varepsilon}{2}\right) \sqrt{\alpha}\left(\frac{1-e^{-x}}{\sqrt{x}}\right) t \sqrt{\ln d}=(1-\varepsilon) \lambda t \sqrt{\ln d}
$$

the graph $G$ satisfies the conditions of the theorem.

We finish with the natural open problem that arises from this work: Can the constant in the upper bound of Reed and Wood [26] be improved to match the lower bound in the present paper? That is, is $f(H) \leqslant(\lambda+o(1)) t \sqrt{\ln d}$ for every graph $H$ with $t$ vertices and average degree $d$ ?

\section{Note Added in Proof}

Following the initial release of this paper, Thomason and Wales [34] announced a solution to the above open problem.

\section{Acknowledgement}

This research was partially completed at the Armenian Workshop on Graphs, Combinatorics and Probability, June 2019. Many thanks to the other organisers and participants. 


\section{References}

[1] Noga Alon and Zoltán Füredi. Spanning subgraphs of random graphs. Graphs Combin., 8(1):91-94, 1992. https://doi.org/10.1007/BF01271712. MR: 1157513.

[2] Béla Bollobás, Paul A. Catlin, and Paul Erdős. Hadwiger's conjecture is true for almost every graph. European J. Combin., 1(3):195-199, 1980. https://doi.org/10.1016/S0195-6698(80)80001-1. MR:593989.

[3] Maria Chudnovsky, Bruce Reed, and Paul Seymour. The edgedensity for $K_{2, t}$ minors. J. Combin. Theory Ser. B, 101(1):18-46, 2011. https://doi.org/10.1016/j.jctb.2010.09.001. MR:2737176.

[4] Endre Csóka, Irene Lo, Sergey Norin, Hehui Wu, and Liana YepreMYAN. The extremal function for disconnected minors. J. Combin. Theory Ser. B, 126:162-174, 2017. https://doi.org/10.1016/j.jctb.2017.04.005. MR: 3667667.

[5] W. Fernandez DE LA Vega. On the maximum density of graphs which have no subcontraction to $K^{s}$. Discrete Math., 46(1):109-110, 1983. https://doi.org/10.1016/0012-365X(83)90280-7. MR:0708172.

[6] Gabriel A. Dirac. Homomorphism theorems for graphs. Math. Ann., 153:69-80, 1964. https://doi.org/10.1007/BF01361708. MR:0160203.

[7] Jасов Fox. Constructing dense graphs with sublinear Hadwiger number. 2011. arXiv: 1108.4953.

[8] Daniel J. Harvey And David R. Wood. Cycles of given size in a dense graph. SIAM J. Discrete Math., 29(4):2336-2349, 2015. https://doi.org/10.1137/15M100852X. MR:3427042.

[9] Daniel J. Harvey and David R. Wood. Average degree conditions forcing a minor. Electron. J. Combin., 23(1):\#P1.42, 2016. https://doi.org/10.37236/5321. MR: 3484747.

[10] Kevin Hendrey, Sergey Norin, and David R. Wood. On the extremal function for sparse minors. In preparation.

[11] Kevin Hendrey And David R. Wood. The extremal function for Petersen minors. J. Combin. Theory Ser. B, 131:220-253, 2018. https://doi.org/10.1016/j.jctb.2018.02.001. MR: 3794147.

[12] Leif K. Jørgensen. Contractions to $K_{8}$. J. Graph Theory, 18(5):431-448, 1994. https://doi.org/10.1002/jgt.3190180502. MR: 1283309.

[13] Rohan Kapadia, Sergey Norin, and Yinguie Qian. Asymptotic density of graphs excluding disconnected minors. 2019. arXiv:1903.03908.

[14] Alexandr V. Kostochka. The minimum Hadwiger number for graphs with a given mean degree of vertices. Metody Diskret. Analiz., 38:37-58, 1982. MR:0713722.

[15] Alexandr V. Kostochka. Lower bound of the Hadwiger number of graphs by their average degree. Combinatorica, 4(4):307-316, 1984. https://doi.org/10.1007/BF02579141. MR: 0779891.

[16] Alexandr V. Kostochka And Noah Prince. On $K_{s, t}$-minors in graphs with given average degree. Discrete Math., 308(19):4435-4445, 2008. 
https://doi.org/10.1016/j.disc.2007.08.041. MR: 2433771.

[17] Alexandr V. Kostochka And NoAh Prince. Dense graphs have $K_{3, t}$ minors. Discrete Math., 310(20):2637-2654, 2010. https://doi.org/10.1016/j.disc.2010.03.026. MR: 2672210.

[18] Alexandr V. Kostochka and Noah Prince. On $K_{s, t}$-minors in graphs with given average degree, II. Discrete Math., 312(24):3517-3522, 2012. https://doi.org/10.1016/j.disc.2012.08.004. MR: 2979480.

[19] Daniela KüHn AND Deryk Osthus. Forcing unbalanced complete bipartite minors. European J. Combin., 26(1):75-81, 2005. https://doi.org/10.1016/j.ejc.2004.02.002. MR: 2101035.

[20] Wolfang Mader. Homomorphieeigenschaften und mittlere Kantendichte von Graphen. Math. Ann., 174:265-268, 1967. https://doi.org/10.1007/BF01364272. MR: 0220616.

[21] Wolfgang Mader. Homomorphiesätze für Graphen. Math. Ann., 178:154-168, 1968. https://doi.org/10.1007/BF01350657. MR: 0229550.

[22] Michael Mitzenmacher and Eli Upfal. Probability and computing. Cambridge University Press, 2005. https://doi.org/10.1017/CB09780511813603.

[23] Joseph SAmuel Myers. Graphs without large complete minors are quasi-random. Combin. Probab. Comput., 11(6):571-585, 2002. https://doi.org/10.1017/S096354830200531X. MR: 1940121.

[24] Joseph SAmuel Myers. The extremal function for unbalanced bipartite minors. Discrete Math., 271(1-3):209-222, 2003. https://doi .org/10.1016/S0012-365X(03)00051-7. MR: 1999544.

[25] Joseph Samuel Myers And Andrew Thomason. The extremal function for noncomplete minors. Combinatorica, 25(6):725-753, 2005. https://doi.org/10.1007/s00493-005-0044-0. MR:2199433.

[26] Bruce Reed and David R. Wood. Forcing a sparse minor. Combin. Probab. Comput., 25(2):300-322, 2016. https://doi.org/10.1017/S0963548315000073. MR: 3455678 .

[27] Bruce Reed AND David R. Wood. 'Forcing a sparse minor'-Corrigendum. Combin. Probab. Comput., 25(2):323, 2016. https://doi.org/10.1017/S0963548315000115. MR: 3455679.

[28] Zi-Xia Song AND RoBin Thomas. The extremal function for $K_{9}$ minors. J. Combin. Theory Ser. B, 96(2):240-252, 2006. https://doi.org/10.1016/j.jctb.2005.07.008. MR:2208353.

[29] Zixia Song. Extremal Functions for Contractions of Graphs. Ph.D. thesis, Georgia Institute of Technology, USA, 2004. MR: 2706190.

[30] Andrew Thomason. An extremal function for contractions of graphs. Math. Proc. Cambridge Philos. Soc., 95(2):261-265, 1984. https://doi .org/10.1017/S0305004100061521. MR:0735367.

[31] Andrew Thomason. The extremal function for complete minors. J. Combin. Theory Ser. B, 81(2):318-338, 2001. https://doi.org/10.1006/jctb.2000.2013. 
MR: 1814910.

[32] Andrew Thomason. Extremal functions for graph minors. In More sets, graphs and numbers, vol. 15 of Bolyai Soc. Math. Stud., pp. 359-380. Springer, Berlin, 2006.

[33] Andrew Thomason. Disjoint unions of complete minors. Discrete Math., 308(19):4370-4377, 2008. https://doi.org/10.1016/j.disc.2007.08.021. MR: 2433863.

[34] Andrew Thomason and Matthew Wales. On the extremal function for graph minors arXiv:1907.11626, 2019. 